\title{
A WEAK-TYPE INEQUALITY FOR DIFFERENTIALLY SUBORDINATE HARMONIC FUNCTIONS
}

\author{
CHANGSUN CHOI
}

\begin{abstract}
Assuming an extra condition, we decrease the constant in the sharp inequality of Burkholder $\mu(|v| \geq 1) \leq 2\|u\|_{1}$ for two harmonic functions $u$ and $v$. That is, we prove the sharp weak-type inequality $\mu(|v| \geq 1) \leq$ $K\|u\|_{1}$ under the assumptions that $|v(\xi)| \leq|u(\xi)|,|\nabla v| \leq|\nabla u|$ and the extra assumption that $\nabla u \cdot \nabla v=0$. Here $\mu$ is the harmonic measure with respect to $\xi$ and the constant $K$ is the one found by Davis to be the best constant in Kolmogorov's weak-type inequality for conjugate functions.
\end{abstract}

Let $D$ be a domain in $\mathbb{R}^{n}$ where $n$ is a positive integer. Let $D_{0}$ be a bounded subdomain of $D$ with $\partial D_{0} \subseteq D$ and $\xi \in D_{0}$. Let $\mu$ be the harmonic measure on $\partial D_{0}$ with respect to $\xi$. Let $K$ be the constant given by

$$
K=\frac{1+\frac{1}{3^{2}}+\frac{1}{5^{2}}+\frac{1}{7^{2}}+\frac{1}{9^{2}}+\cdots}{1-\frac{1}{3^{2}}+\frac{1}{5^{2}}-\frac{1}{7^{2}}+\frac{1}{9^{2}}-\cdots} .
$$

Theorem. If $u$ and $v$ are harmonic functions on $D$ such that

$$
|v(\xi)| \leq|u(\xi)|
$$

$|\nabla v| \leq|\nabla u|$ on $D$

then

$$
\mu(|v| \geq 1) \leq K \int_{\partial D_{0}}|u| d \mu .
$$

Remarks. 1. The constant $K$ was discovered by Davis [6]. He showed that $K$ is the best constant in Kolmogorov's weak-type inequality for conjugate functions [9] or, equivalently, for the special case of the inequality above in which $D$ is the open unit disk of $\mathbb{R}^{2}, D_{0}$ is an open disk with center 0 and radius $r<1, \xi=0, v(0)=0$, and $u$ and $v$ are harmonic in $D$ and satisfy the Cauchy-Riemann equations. Also, see Baernstein [2] for related sharp inequalities.

2. Dropping the classical conjugacy condition and working in $\mathbb{R}^{n}$, Burkholder [4] proved the sharp inequality

$$
\mu(|u|+|v| \geq 1) \leq 2 \int_{\partial D_{0}}|u| d \mu
$$

for harmonic functions $u$ and $v$ that satisfy the assumptions (i) and (ii) of the theorem. In fact, he proved his inequality for Hilbert-space valued $u$ and $v$. In

Received by the editors October 7, 1995

1991 Mathematics Subject Classification. Primary 31B05, 31B15; Secondary 42A50, 60G42.

Key words and phrases. Harmonic functions, harmonic measure, differential subordination, weak-type inequality, Burkholder's inequality, Kolmogorov's inequality, Davis's constant.

(C)1998 American Mathematical Society 
Example 13.1 of [5], he showed that 2 is the best constant even for the inequality $\mu(|v| \geq 1) \leq 2\|u\|_{1}$ where $\|u\|_{p}^{p}=\sup _{D_{0}} \int_{\partial D_{0}}|u|^{p} d \mu$.

3. Using (i) and (ii), Burkholder [4] also proved that $\|v\|_{p} \leq\left(p^{*}-1\right)\|u\|_{p}$ for $1<p<\infty$ where $p^{*}=\max \{p, p /(p-1)\}$. It is not yet known whether the constant $p^{*}-1$ is best possible in this setting. However, using (i), (ii), and the extra assumption (iii), Bañuelos and Wang [3] proved the inequality $\|v\|_{p} \leq$ $\cot \left(\pi / 2 p^{*}\right)\|u\|_{p}$ for $1<p<\infty$. This is a sharp inequality since it is already sharp in the classical M. Riesz case [11] in which $v(0)=0$ and $v$ is the harmonic function conjugate to $u$ on the open unit disk of the plane (see Pichorides [10] and the independent work of Brian Cole that is described in Gamelin [7]).

Outline of the proof of the theorem. Consider the function $V$ on $\mathbb{R}^{2}$ given by

$$
V(x, y)= \begin{cases}-K|x| & \text { if }|y|<1 \\ 1-K|x| & \text { if }|y| \geq 1\end{cases}
$$

We observe that

$$
\mu(|v| \geq 1)-K \int_{\partial D_{0}}|u| d \mu=\int_{\partial D_{0}} V(u, v) d \mu .
$$

The following lemma will be proved later:

Main Lemma. There is a continuous function $U$ on $\mathbb{R}^{2}$ such that

$$
\begin{aligned}
& \text { (a) } \quad V \leq U \text { on } \mathbb{R}^{2}, \\
& \text { (b) } U(u, v) \text { is superharmonic on } D, \\
& \text { (c) } \quad U(x, y) \leq 0 \quad \text { if }|x| \geq|y| .
\end{aligned}
$$

Then from (a) and (b) we get

$$
\int_{\partial D_{0}} V(u, v) d \mu \leq \int_{\partial D_{0}} U(u, v) d \mu \leq U(u(\xi), v(\xi))
$$

because $\mu$ is the harmonic measure on $\partial D_{0}$ with respect to $\xi$. Finally, (c) and the assumption (i) imply that $U(u(\xi), v(\xi)) \leq 0$, which proves the theorem.

Before we prove the lemma we define another function $W$ on $\mathbb{R}^{2}$ and establish some properties of $W$. We will use basic facts about harmonic functions, which can be found in [1] and [8].

Put $H=\{(\alpha, \beta): \beta>0\}, S=\{(x, y):|y|<1\}$ and $S^{+}=\{(x, y) \in S: x>0\}$. Also, put $(x, y)=x+i y=z, \mathfrak{I}(x+i y)=y,(\alpha, \beta)=\alpha+i \beta=\zeta$, and define a function $\mathcal{W}$ on $H$ by

$$
\mathcal{W}(\alpha, \beta)=\mathcal{W}(\zeta)=\frac{2}{\pi^{2}} \int_{-\infty}^{\infty} \frac{\beta|\log | t||}{(\alpha-t)^{2}+\beta^{2}} d t .
$$

Observe that $\mathcal{W}$ is the harmonic function on $H$ that vanishes as $\beta \rightarrow \infty$, and satisfies

$$
\lim _{(\alpha, \beta) \rightarrow(t, 0)} \mathcal{W}(\alpha, \beta)=\frac{2}{\pi}|\log | t|| \quad \text { if } t \neq 0
$$


Using $\pi^{2} / 8=\sum_{k=0}^{\infty}(2 k+1)^{-2}$, we have that

$$
\begin{aligned}
\mathcal{W}(0,1) & =\frac{4}{\pi^{2}} \int_{0}^{\infty} \frac{|\log t|}{t^{2}+1} d t \\
& =\frac{4}{\pi^{2}} \int_{-\infty}^{\infty} \frac{|s| e^{s}}{e^{2 s}+1} d s \\
& =\frac{8}{\pi^{2}} \int_{0}^{\infty} s e^{-s} \sum_{k=0}^{\infty}\left(-e^{-2 s}\right)^{k} d s \\
& =\frac{8}{\pi^{2}} \sum_{k=0}^{\infty} \frac{(-1)^{k}}{(2 k+1)^{2}}=\frac{1}{K} .
\end{aligned}
$$

Consider the conformal map $\varphi$ on $S$ given by

$$
\varphi(z)=i e^{\pi z / 2}=\exp \frac{\pi}{2}(z+i) .
$$

Observe that $\varphi(i)=-1, \varphi(-i)=1, \varphi(-\infty+i y)=0, \varphi(\infty+i y)=\infty$ and $\varphi(0)=i$. Hence $\varphi$ maps the strip $S$ onto the upper half plane $H$. Define $W: \mathbb{R}^{2} \rightarrow \mathbb{R}$ by

$$
W(x, y)= \begin{cases}|x| & \text { if }|y| \geq 1 \\ \mathcal{W}(\varphi(x, y)) & \text { if }|y|<1\end{cases}
$$

and notice that the restriction of $W$ to $S$ is harmonic since this restriction is the real part of an analytic function. For $x_{0} \in \mathbb{R}$ we have $\varphi\left(x_{0}, \pm 1\right)= \pm e^{\pi x_{0} / 2} \neq 0$, thus

$$
\lim _{\substack{(x, y) \rightarrow\left(x_{0}, \pm 1\right) \\(x, y) \in S}} W(x, y)=\frac{2}{\pi}|\log | \varphi\left(x_{0}, \pm 1\right)|=| x_{0} \mid=W\left(x_{0}, \pm 1\right) .
$$

Hence $W$ is continuous on $\mathbb{R}^{2}$ as is the function $U$ defined by

$$
U(x, y)=1-K W(x, y) \quad \text { for }(x, y) \in \mathbb{R}^{2} .
$$

Lemma 1. If $(x, y) \in S$, then $W(x, y)=W(-x, y)=W(x,-y)$ and

$$
W_{x}(0, y)=W_{y}(x, 0)=W_{x y}(x, 0)=W_{x y}(0, y)=0 .
$$

Proof. In (1) we use the change of variable $t=-s$ to get $\mathcal{W}(-\alpha, \beta)=\mathcal{W}(\alpha, \beta)$. Also, in the reformulation of $\mathcal{W}$

$$
\mathcal{W}(\zeta)=\frac{4}{\pi^{2}} \mathfrak{I} \int_{0}^{\infty} \frac{\zeta|\log t|}{t^{2}-\zeta^{2}} d t
$$

we use the change of variable $t=1 / s$ to get $\mathcal{W}(1 / \bar{\zeta})=\mathcal{W}(\zeta)$. With $\varphi(x, y)=\zeta=$ $\alpha+i \beta$ we get $\varphi(-x, y)=1 / \bar{\zeta}$ and $\varphi(x,-y)=-\alpha+i \beta$. The symmetry of $W$ and the rest of the lemma follow.

Lemma 2. $\lim _{\substack{x, y) \in S \\ x \rightarrow \infty}}[W(x, y)-x]=0$.

Proof. $\varphi(x, y)=\zeta$ we have $x=\frac{2}{\pi} \log |\zeta|$, hence $x \rightarrow \infty$ if and only if $|\zeta| \rightarrow \infty$ and the lemma is equivalent to

$$
\lim _{|\zeta| \rightarrow \infty}\left[\mathcal{W}(\zeta)-\frac{2}{\pi} \log |\zeta|\right]=0 .
$$


On $H$, the harmonic function $\zeta \mapsto \frac{2}{\pi} \log |\zeta|$ can be represented by its Poisson integral. Therefore, by (1),

$$
\begin{aligned}
\mathcal{W}(\zeta)-\frac{2}{\pi} \log |\zeta| & =\frac{2}{\pi^{2}} \int_{-\infty}^{\infty}\left[\frac{\beta|\log | t||}{(\alpha-t)^{2}+\beta^{2}}-\frac{\beta \log |t|}{(\alpha-t)^{2}+\beta^{2}}\right] d t \\
& =\frac{4}{\pi^{2}} \int_{-1}^{1} \frac{\beta|\log | t||}{(\alpha-t)^{2}+\beta^{2}} d t \rightarrow 0 \quad \text { as }|\zeta| \rightarrow \infty
\end{aligned}
$$

which proves (2), hence the lemma.

Lemma 3. $\lim _{\substack{x \rightarrow y) \in S \\ x \rightarrow \infty}} W_{x x}(x, y)=\lim _{(x, y) \in S}{ }_{x \rightarrow \infty}^{\infty} W_{x y}(x, y)=0$.

Proof. Consider the continuous function $G$ on $\overline{S^{+}}$given by $G(x, y)=W(x, y)-x$. Observe that $G$ is harmonic on $S^{+}$and $G(x, \pm 1)=0$.

We consider a conformal map $\psi$ given by $\psi(z) \sin \left(\frac{\pi}{2} i z\right)=-1$. One checks that $\psi\left(\frac{2}{\pi} \log (1+\sqrt{2})\right)=i, \psi(0)=\infty, \psi(-i)=-1, \psi(\infty)=0$ and $\psi(i)=1$. Thus $S^{+}$ is mapped onto $H$ under $\psi$.

We define a harmonic function $F(\alpha, \beta)$ on $H$ by $G=F \circ \psi$. For $|t|<1$ we have

$$
\lim _{(\alpha, \beta) \rightarrow(t, 0)} F(\alpha, \beta)=0 .
$$

Indeed, we have from Lemma 2 that if $t=0$, then

$$
0=\lim _{\substack{x \rightarrow \infty \\(x, y) \in S}} G(x, y)=\lim _{(\alpha, \beta) \rightarrow(0,0)} F(x, y) .
$$

Also, for $t \neq 0$, since $\psi^{-1}(t, 0)=(c, \pm 1)$ for some $c$ and $G(c, \pm 1)=0$, the limit (3) follows from the continuity of $G$.

Applying the Schwarz reflection principle, we see that the functions $F_{\alpha}(\alpha, \beta)$, $F_{\beta}(\alpha, \beta), F_{\alpha \alpha}(\alpha, \beta), F_{\alpha \beta}(\alpha, \beta)$ and $F_{\beta \beta}(\alpha, \beta)$ tend to certain limits as $(\alpha, \beta)$ tends to $(0,0)$.

Now from the basic identities

$$
|\cos i z|^{2}=\sinh ^{2} x+\cos ^{2} y \text { and }|\sin i z|^{2}=\sinh ^{2} x+\sin ^{2} y
$$

we observe that

$$
\lim _{x \rightarrow \infty} \cos \frac{\pi}{2} i z=\lim _{x \rightarrow \infty} \sin \frac{\pi}{2} i z=\infty \quad \text { and } \quad \lim _{x \rightarrow \infty}\left|\tan \frac{\pi}{2} i z\right|=1 .
$$

Differentiating $\psi(z) \sin \left(\frac{\pi}{2} i z\right)=-1$, we get

$$
\begin{gathered}
\psi^{\prime}(z) \sin \frac{\pi}{2} i z+\frac{\pi}{2} i \psi(z) \cos \frac{\pi}{2} i z=0, \\
\psi^{\prime \prime}(z) \sin \frac{\pi}{2} i z+\pi i \psi^{\prime}(z) \cos \frac{\pi}{2} i z+\frac{\pi^{2}}{4} \psi(z) \sin \frac{\pi}{2} i z=0 .
\end{gathered}
$$

Hence, if $z=(x, y) \in S^{+}$and $|z| \rightarrow \infty$, then $\lim \psi(z)=\lim \psi^{\prime}(z)=\lim \psi^{\prime \prime}(z)=0$.

Writing $\psi(x+i y)=\alpha(x, y)+i \beta(x, y)$, we see that as $(x, y) \in S^{+}$and $x \rightarrow \infty$ all the functions $\alpha, \beta, \alpha_{x}, \beta_{x}, \alpha_{x x}, \beta_{x x}$ tend to 0 because $\psi^{\prime}=\alpha_{x}+i \beta_{x}$ and $\psi^{\prime \prime}=\alpha_{x x}+i \beta_{x x}$.

From the Cauchy-Riemann equations we have $\alpha_{y}=-\beta_{x}$ and $\beta_{y}=\alpha_{x}$, so $\alpha_{x y}=$ $-\beta_{x x}$ and $\beta_{x y}=\alpha_{x x}$. Thus, using the chain rule and omitting the argument $(x, y)$, 
we have

$$
\begin{aligned}
G_{x}= & \alpha_{x} F_{\alpha}(\alpha, \beta)+\beta_{x} F_{\beta}(\alpha, \beta), \\
G_{x x}= & \left(\alpha_{x}\right)^{2} F_{\alpha \alpha}(\alpha, \beta)+\left(\beta_{x}\right)^{2} F_{\beta \beta}(\alpha, \beta)+2 \alpha_{x} \beta_{x} F_{\alpha \beta}(\alpha, \beta) \\
& +a_{x x} F_{\alpha}(\alpha, \beta)+\beta_{x x} F_{\beta}(\alpha, \beta), \\
G_{x y}= & -\alpha_{x} \beta_{x} F_{\alpha \alpha}(\alpha, \beta)+\alpha_{x} \beta_{x} F_{\beta \beta}(\alpha, \beta)+\left[\left(\alpha_{x}\right)^{2}-\left(\beta_{x}\right)^{2}\right] F_{\alpha \beta}(\alpha, \beta) \\
& -\beta_{x x} F_{\alpha}(\alpha, \beta)+\alpha_{x x} F_{\beta}(\alpha, \beta) .
\end{aligned}
$$

It follows that

$$
\lim _{\substack{x \rightarrow \infty \\(x, y) \in S^{+}}} G_{x x}(x, y)=\lim _{\substack{x \rightarrow \infty \\(x, y) \in S^{+}}} G_{x y}(x, y)=0 .
$$

This proves the lemma because $G_{x x}=W_{x x}$ and $G_{x y}=W_{x y}$.

Lemma 4. Consider the function $A$ on $H$ given by

Then we have

$$
A(x, y)=\frac{1}{\pi} \int_{-1}^{1} \frac{y|t|}{(x-t)^{2}+y^{2}} d t
$$

$$
\liminf _{(x, y) \rightarrow(0,0)} A_{x x}(x, y) \geq 0 \quad \text { and } \quad \limsup _{\substack{(x, y) \rightarrow(0,0) \\ x>0}} A_{x y}(x, y) \leq 0 .
$$

Proof. Differentiating under the integral sign and then integrating by parts, we get

$$
\begin{aligned}
\pi A_{x}(x, y) & =\int_{0}^{1} t \frac{\partial}{\partial t}\left[\frac{y}{(x+t)^{2}+y^{2}}-\frac{y}{(x-t)^{2}+y^{2}}\right] d t \\
& =\frac{y}{(x+1)^{2}+y^{2}}-\frac{y}{(x-1)^{2}+y^{2}} \\
& -\int_{0}^{1}\left[\frac{y}{(x+t)^{2}+y^{2}}-\frac{y}{(x-t)^{2}+y^{2}}\right] d t .
\end{aligned}
$$

Differentiating under the integral again, we get

$$
\begin{aligned}
\pi A_{x x}(x, y)= & -\frac{2(x+1) y}{\left[(x+1)^{2}+y^{2}\right]^{2}}+\frac{2(x-1) y}{\left[(x-1)^{2}+y^{2}\right]^{2}} \\
& -\frac{y}{(x+1)^{2}+y^{2}}-\frac{y}{(x-1)^{2}+y^{2}}+\frac{2 y}{x^{2}+y^{2}}
\end{aligned}
$$

and

$$
\begin{aligned}
\pi A_{x y}(x, y)= & \frac{(x+1)^{2}-y^{2}}{\left[(x+1)^{2}+y^{2}\right]^{2}}-\frac{(x-1)^{2}-y^{2}}{\left[(x-1)^{2}+y^{2}\right]^{2}} \\
& +\frac{x-1}{(x-1)^{2}+y^{2}}+\frac{x+1}{(x+1)^{2}+y^{2}}-\frac{2 x}{x^{2}+y^{2}} .
\end{aligned}
$$

Since $y>0$ we have

$$
\liminf _{(x, y) \rightarrow(0,0)} A_{x x}(x, y)=\frac{1}{\pi} \liminf _{(x, y) \rightarrow(0,0)} \frac{2 y}{x^{2}+y^{2}} \geq 0 .
$$

Also,

$$
\limsup _{\substack{(x, y) \rightarrow(0,0) \\ x>0}} A_{x y}(x, y)=\frac{1}{\pi} \limsup _{\substack{(x, y) \rightarrow(0,0) \\ x>0}}\left(-\frac{2 x}{x^{2}+y^{2}}\right) \leq 0 .
$$




\section{Lemma 5.}

$$
\liminf _{\substack{(x, y) \rightarrow(0,-1) \\(x, y) \in S}} W_{x x}(x, y) \geq 0 \quad \text { and } \quad \limsup _{\substack{(x, y) \rightarrow(0,-1) \\(x, y) \in S \\ x>0}} W_{x y}(x, y) \leq 0 .
$$

Proof. Let $A$ be the function given in Lemma 4. Define $B(x, y)$ on $S$ by

$$
B(x, y)=W(x, y)-A(x, y+1) .
$$

Observe that $B$ is harmonic on $S$ and if $\left|x_{0}\right|<1$, then

$$
\lim _{(x, y) \rightarrow\left(x_{0},-1\right)} B(x, y)=\left|x_{0}\right|-\lim _{(x, y) \rightarrow\left(x_{0}, 0\right)} A(x, y)=0 .
$$

Applying the Schwarz reflection principle we get a harmonic extension $B^{*}$ of $B$ over $S \cup\{(x,-1):|x|<1\} \cup\{(x, y): x \in \mathbb{R},-3<y<-1\}$.

Note that $B^{*}(x,-1)=0$ for $|x|<1$. Thus $B_{x x}^{*}(0,-1)=0$. Both $W$ and $A$ are symmetric with respect to $y$-axis, hence so is $B^{*}$. Thus $B_{x y}^{*}(0,-1)=0$. From Lemma 4 and the limit

$$
\lim _{(x, y) \rightarrow(0,-1)} B_{x x}(x, y)=B_{x x}^{*}(0,-1)=0
$$

we get

$$
\liminf _{\substack{(x, y) \rightarrow(0,-1) \\(x, y) \in S}} W_{x x}(x, y)=\lim _{\substack{(x, y) \rightarrow(0,-1) \\(x, y) \in S}} B_{x x}(x, y)+\liminf _{\substack{(x, y) \rightarrow(0,0) \\(x, y) \in H}} A_{x x}(x, y) \geq 0 .
$$

The inequality about limsup $W_{x y}$ is obtained similarly.

Lemma 6. If $x_{0}>0$, then

$$
\lim _{\substack{(x, y) \rightarrow\left(x_{0},-1\right) \\(x, y) \in S}} W_{x x}(x, y)=0 \quad \text { and } \quad \lim _{\substack{(x, y) \rightarrow\left(x_{0},-1\right) \\(x, y) \in S}} W_{x y}(x, y) \leq 0 .
$$

Proof. Let $x_{0}>0$. Define a harmonic function $C$ on $S$ by $C(x, y)=W(x, y)-x$. Observe that for $x_{0} \geq 0$ we have

$$
\lim _{(x, y) \rightarrow\left(x_{0},-1\right)} C(x, y)=0 .
$$

We apply the Schwarz reflection principle to get a harmonic extension $C^{*}$ of $C$ over $S \cup\{(x,-1): x>0\} \cup\{(x, y): x \in \mathbb{R},-3<y<-1\}$.

If $x>0$, then $C^{*}(x,-1)=0$, hence $C_{x x}^{*}(x,-1)=0$ and

$$
\lim _{\substack{(x, y) \rightarrow\left(x_{0},-1\right) \\(x, y) \in S}} W_{x x}(x, y)=\lim _{(x, y) \rightarrow\left(x_{0},-1\right)} C_{x x}(x, y)=C_{x x}^{*}\left(x_{0},-1\right)=0
$$

which proves the first part of the lemma.

For the second part of the lemma we apply the Maximum Principle to $C_{x y}^{*}$ over $\Omega=\{(x, y): x>0$ and $-2<y<0\}$ to get $C_{x y}^{*}\left(x_{0},-1\right) \leq 0$ which yields

$$
\lim _{\substack{(x, y) \rightarrow\left(x_{0},-1\right) \\(x, y) \in S}} W_{x y}(x, y)=\lim _{\substack{(x, y) \rightarrow\left(x_{0},-1\right) \\(x, y) \in S}} C_{x y}(x, y)=C_{x y}^{*}\left(x_{0},-1\right) \leq 0 .
$$

Now we will check the boundary conditions of the Maximum Principle. For $-3<y<-1$ we have $C^{*}(x, y)=-C(x,-y-2)$, hence

$$
C_{x y}^{*}(x, y)=C_{x y}(x,-y-2)=W_{x y}(x,-y-2) .
$$


From Lemma 1 we get $C_{x y}^{*}(0, y)=0$ if $0<|y+1|<1$. Also, from Lemma 1 $W_{x y}(x, 0)=0$. Thus if $x_{1}>0$ and $\left|y_{0}+1\right|=1$, then

$$
\limsup _{\substack{(x, y) \rightarrow\left(x_{1}, y_{0}\right) \\(x, y) \in \Omega}} C_{x y}^{*}(x, y)=C_{x y}^{*}\left(x_{1}, y_{0}\right)=W_{x y}\left(x_{1}, 0\right)=0 .
$$

And using Lemma 3, we have

$$
\limsup _{\substack{x \rightarrow \infty \\(x, y) \in \Omega}} C_{x y}^{*}(x, y)=\lim _{\substack{x \rightarrow \infty \\(x, y) \in S}} W_{x y}(x, y)=0
$$

because $C_{x y}^{*}(x, y)=W_{x y}(x,-y-2)$ for $-2<y<-1$ and $C_{x y}^{*}$ is continuous on $\Omega$. Finally, the second part of Lemma 5 implies that

$$
\limsup _{\substack{(x, y) \rightarrow(0,-1) \\(x, y) \in \Omega}} C_{x y}^{*}(x, y)=\limsup _{\substack{(x, y) \rightarrow(0,-1) \\(x, y) \in S \\ x>0}} W_{x y}(x, y) \leq 0 .
$$

This checks all the boundary conditions and finishes the proof of the lemma.

Lemma 7. $W_{x x} \geq 0$ on $S$.

Proof. We apply the Maximum Principle to the harmonic function $-W_{x x}$ over $S$.

Observe, from Lemma 1 , that $W_{x x}(-x, y)=W_{x x}(x,-y)=W_{x x}(x, y)$.

It remains to check the boundary conditions. The first part of Lemma 3 implies

$$
\limsup _{\substack{|x| \rightarrow \infty \\(x, y) \in S}}\left[-W_{x x}(x, y)\right]=-\lim _{\substack{|x| \rightarrow \infty \\(x, y) \in S}} W_{x x}(x, y)=0 .
$$

For $x_{0} \neq 0$, the first part of Lemma 6 gives

$$
\limsup _{\substack{(x, y) \rightarrow\left(x_{0}, \pm 1\right) \\(x, y) \in S}}\left[-W_{x x}(x, y)\right]=-\lim _{\substack{(x, y) \rightarrow\left(x_{0},-1\right) \\(x, y) \in S}} W_{x x}(x, y)=0
$$

Finally, the first part of Lemma 5 gives

$$
\limsup _{\substack{(x, y) \rightarrow(0, \pm 1) \\(x, y) \in S}}\left[-W_{x x}(x, y)\right]=-\liminf _{\substack{(x, y) \rightarrow(0,-1) \\(x, y) \in S}} W_{x x}(x, y) \leq 0 .
$$

This proves the lemma.

Lemma 8. $W_{x y} \leq 0$ on $\Omega=\{(x, y): x>0$ and $-1<y<0\}$.

Proof. Note that $W_{x y}$ is harmonic on $\Omega$ and it is continuous on $S$. For $x_{0}>0$, Lemma 1 implies

$$
\limsup _{\substack{(x, y) \rightarrow\left(x_{0}, 0\right) \\(x, y) \in \Omega}} W_{x y}\left(x_{0}, 0\right)=W_{x y}\left(x_{0}, 0\right)=0
$$

and the second part of Lemma 6 implies

$$
\limsup _{\substack{(x, y) \rightarrow\left(x_{0},-1\right) \\(x, y) \in \Omega}} W_{x y}(x, y)=\lim _{\substack{(x, y) \rightarrow\left(x_{0},-1\right) \\(x, y) \in S}} W_{x y}(x, y) \leq 0 .
$$

Let $-1<y_{0} \leq 0$. Lemma 1 gives

$$
\limsup _{\substack{(x, y) \rightarrow\left(0, y_{0}\right) \\(x, y) \in \Omega}} W_{x y}(x, y)=W_{x y}\left(0, y_{0}\right)=0 .
$$


Also, from the second part of Lemma 5,

$$
\limsup _{\substack{(x, y) \rightarrow(0,-1) \\(x, y) \in \Omega}} W_{x y}(x, y)=\limsup _{\substack{(x, y) \rightarrow(0,-1) \\(x, y) \in S \\ x>0}} W_{x y}(x, y) \leq 0,
$$

and from the second part of Lemma 3

$$
\limsup _{\substack{x \rightarrow \infty \\(x, y) \in \Omega}} W_{x y}(x, y)=\lim _{\substack{x \rightarrow \infty \\(x, y) \in S}} W_{x y}(x, y)=0 .
$$

Therefore we can apply the Maximum Principle and the lemma follows.

Proof of Main Lemma. We have defined the continuous function $U$ on $\mathbb{R}^{2}$. It remains to show the properties (a), (b) and (c) of the function $U$.

Proof of (a). By the definitions we have $U(x, y)=V(x, y)$ if $|y| \geq 1$. Also, $W(0,0)$ $=\mathcal{W}(\varphi(0,0))=\mathcal{W}(0,1)=\frac{1}{K}$. Thus, if $|y|<1$, then

$$
U(x, y)=1-K W(x, y)=-K[W(x, y)-W(0,0)] .
$$

Hence the property (a) follows if $-K|x| \leq-K[W(x, y)-W(0,0)]$ on $S$. By the symmetry of $W$ it suffices to show

$$
E(x, y) \leq 0 \quad \text { if } \quad(x, y) \in \overline{S^{+}}
$$

where $E(x, y)=W(x, y)-W(0,0)-|x|$. Using Lemma 2 we have

$$
\limsup _{\substack{x \rightarrow \infty \\(x, y) \in S^{+}}} E(x, y)=-W(0,0)<0 .
$$

Also $E(x, \pm 1)=-W(0,0)<0$ for $x \geq 0$. Since $W$ is harmonic on $S$ we have $W_{x x}+W_{y y}=0$ thus $W_{y y}=-W_{x x} \leq 0$ on $S$ by Lemma 7 . Hence, for $|y|<1$, $E_{y y}(0, y)=W_{y y}(0, y) \leq 0$ and $E(0, y)$ is a concave function on $y$. But $E_{y}(0,0)=$ $W_{y}(0,0)=0$ from Lemma 1 . Thus $E(0, y) \leq E(0,0)=0$ for $|y|<1$. Because $E$ is continuous on $\overline{S^{+}}$the Maximum Principle proves the inequality (5), hence the property (a).

Proof of (b). By (4) the property (b) becomes

$$
W(u, v) \text { is subharmonic on } D \text {. }
$$

Arguing similarly as in the proof of (a) one gets

$$
W(x, y) \geq|x| \text { if }|y|<1 .
$$

Now we put $w=W(u, v)$ on $D$. When $|v|>1$ clearly $w=|u|$ is subharmonic because $u$ is harmonic. When $|v|<1$, writing $W_{x}$ for $W_{x}(u, v)$ etc., we have

$$
\begin{aligned}
\Delta w & =W_{x x}|\nabla u|^{2}+W_{y y}|\nabla v|^{2}+2 W_{x y} \nabla u \cdot \nabla v+W_{x} \Delta u+W_{y} \Delta v \\
& =W_{x x}\left(|\nabla u|^{2}-|\nabla v|^{2}\right) \geq 0,
\end{aligned}
$$

hence $w$ is subharmonic. In the above we used the assumptions (ii) and (iii), Lemma 7 and the harmonicity of $u$ and $v$. When $|v|=1$ at $\eta \in D$ we have, for all small $r>0$, that

$$
\operatorname{Avg}(w ; \eta, r) \geq \operatorname{Avg}(|u| ; \eta, r) \geq|u(\eta)|=w(\eta)
$$


thus $w$ is subharmonic at $\eta$. In the above we used the inequality (7). Also $\operatorname{Avg}(w ; \eta, r)$ is the average of $w$ over the ball $\{\lambda \in D:|\lambda-\eta|<r\}$ with respect to the Lebesgue measure in $\mathbb{R}^{n}$. This proves (6), hence (b).

Proof of (c). By (4) the property (c) of $U$ follows from

$$
W(x, y) \geq W(0,0) \quad \text { if } \quad|x| \geq|y| .
$$

Let $I_{0}=[0, \infty)$ and for $-1 \leq a<0$, put $I_{a}=\left[0,-\frac{1}{a}\right)$. Define $\Phi_{a}$ by $\Phi_{a}(t)=$ $W(t, a t)$ for $t \in I_{a}$. Then for $t$ in the interior of $I_{a}$

$$
\Phi_{a}^{\prime}(t)=W_{x}(t, a t)+a W_{y}(t, a t)
$$

and

$$
\begin{aligned}
\Phi_{a}^{\prime \prime}(t) & =W_{x x}(t, a t)+a^{2} W_{y y}(t, a t)+2 a W_{x y}(t, a t) \\
& =\left(1-a^{2}\right) W_{x x}(t, a t)+2 a W_{x y}(t, a t) \\
& \geq 0
\end{aligned}
$$

because $W$ is harmonic, $W_{x x}(t, a t) \geq 0$ by Lemma 7 and because $W_{x y}(t, a t) \leq 0$ by Lemma 8 . Observe that $\Phi_{a}^{\prime}(0)=W_{x}(0,0)+a W_{y}(0,0)=0$ by Lemma 1 . Hence $\Phi_{a}(t) \geq \Phi_{a}(0)$ for $t \in I_{a}$. Thus $W(t, a t) \geq W(0,0)$ if $-1 \leq a \leq 0$ and $t \in I_{a}$. But $\{(x, y): x \geq-y$ and $-1<y \leq 0\}=\left\{(t, a t):-1 \leq a \leq 0\right.$ and $\left.t \in I_{a}\right\}$. Using the symmetry of $W$ we have

$$
W(x, y) \geq W(0,0) \quad \text { if } \quad|x| \geq|y| \quad \text { and } \quad|y|<1 .
$$

Also, if $|x| \geq|y|$ and $|y| \geq 1$, then

$$
W(x, y)=|x| \geq 1>\frac{1}{K}=W(0,0) .
$$

This proves (8), hence (c).

\section{ACKNOWLEDGEMENT}

It gives me great pleasure to thank Professor D. L. Burkholder who gave me valuable references as well as the problem. I also would like to thank Professor J.-M. Wu who illustrated the usefulness of the Schwarz reflection principle. Finally, I would like to thank Hilda K. Britt who did excellent typing.

\section{REFERENCES}

1. S. Axler et al., Harmonic function theory, Springer-Verlag, 1992. MR 93f:31001

2. A. Baernstein II, Some sharp inequalities for conjugate functions, Indiana Univ. Math. J. 27 (1978), 833-852. MR 80g:30022

3. R. Bañuelos and G. Wang, Sharp inequalities for martingales with applications to the Beurling-Ahlfors and Riesz transformations, Duke Math. J. 80 (1995), 575-600. MR 96k:60108

4. D. L. Burkholder, Differential subordination of harmonic functions and martingales, Harmonic Analysis and Partial Differential Equations (El Escorial, 1987), Lecture Notes in Mathematics 1384 (1989), 1-23. MR 90k:31004

5. D. L. Burkholder, Strong differential subordination and stochastic integration, Ann. Probab. 22 (1994), 995-1025. MR 95k:60085

6. B. Davis, On the weak type $(1,1)$ inequality for conjugate functions, Proc. Amer. Math. Soc. 44 (1974), 307-311. MR 50:879

7. T. W. Gamelin, Uniform algebras and Jensen measures, Cambridge University Press, London, 1978.

8. Y. Katznelson, An introduction to harmonic analysis, Dover Publications, 1976. MR 54:10976 
9. A. N. Kolmogorov, Sur les fonctions harmoniques conjugées et les séries de Fourier, Fund. Math. 7 (1925), 24-29.

10. S. K. Pichorides, On the best values of the constants in the theorems of M. Riesz, Zygmund and Kolmogorov, Studia Math. 44 (1972), 165-179. MR 47:702

11. M. Riesz, Sur les fonctions conjugées, Math. Z. 27 (1927), 218-244.

Department of Mathematics, University of Illinois, 273 Altgeld Hall, 1409 West Green Street, Urbana, Illinois 61801

Current address: Department of Mathematics, KAIST Taejon, 305-701 Korea

E-mail address: cschoi@math.kaist.ac.kr 